system at which we were able to target attempts at improvements. We would like to point out that we have a policy for telephoning all important results to the clinicians directly, so by-passing the written report.

In future, the computerisation of the microbiology service with terminals in all clinical areas will lead to a more efficient turnaround time.

1 Pennington GW. Laboratory accreditation and audit in pathology. The maintenance of standards of pathology in
Britain: the current position. J Clin Pathol 1989;42:561-2.

2 Hoffman G. Laboratory accreditation and audit in pathology: Medical audit in the United States. J Clin Pathol 1989;42:562.

\title{
Comparative yield of Salmonella typhi from blood and bone marrow cultures in patients with fever of unknown origin
}

\author{
B J Farooqui, $M$ Khurshid, $M$ K Ashfaq, $M$ Ata Khan
}

\begin{abstract}
Over three years, a comparative study on 100 selected patients with fever of unknown origin was undertaken to determine the yield of Salmonella typhi from their blood and bone marrow cultures. The results indicate that in patients who had an infection with $S$ typhi the organism was isolated from the bone marrow in all of them and from the blood in only $66 \%$. This suggests that bone marrow cultures may be attempted when blood cultures are negative for bacterial growth after three to four days of incubation.
\end{abstract}

Infection with Salmonella typhi is one of the commonest causes of typhoid fever in Karachi, Pakistan. Over three years, we processed 21356 blood samples from patients suspected of typhoid fever. The average isolation rate for Salmonella from these blood cultures was $5 \cdot 15 \%$. Further subtyping of these Salmonella species showed an incidence of $S$ typhi $83 \%$, $S$ paratyphi $A \quad 11 \cdot 8 \%, S$ paratyphi $B \quad 4 \cdot 1 \%$ and $S$ paratyphi $C 0.9 \% .{ }^{1}$ Definitive diagnosis is made on the basis of isolation of the organism from blood. ${ }^{2}$ Without antibiotics blood cultures are positive in over $80 \%$ of the patients seen in the first week of illness. ${ }^{3}$ Twenty to $30 \%$ of untreated patients have positive blood cultures as late as the third week of illness.

Antimicrobial treatment diminishes the possibility of recovery of the organism in blood. In patients who have been treated cultures of supplementary sites such as bone marrow may be a better source of organisms than blood. ${ }^{4}$ We conducted a study on 100 selected patients whose blood and bone marrow were cultured for $S$ typhi to determine the yield of $S$ typhi from these two sites.

\section{Methods}

Blood and bone marrow samples were collected from 100 patients who had fever of unknown origin. Each sample was collected in two blood culture bottles containing brain heart infusion and thioglycollate broth, respectively.

About $5 \mathrm{ml}$ of venous blood was inoculated in each bottle containing $45 \mathrm{ml}$ of broth; similarly, $0.5 \mathrm{ml}-1.0 \mathrm{ml}$ of bone marrow was collected in each bottle containing $45 \mathrm{ml}$ of broth. Bottles were sent immediately to the laboratory and incubated at $37^{\circ} \mathrm{C}$ for seven days. Each bottle was examined daily and subcultured on to blood agar and MacConkey's media after 24, 48, and 72 hours, and on the seventh day of incubation.

Non-lactosing fermenting colonies from MacConkey's medium were tested for $S$ typhi by slide agglutination test with specific antisera. ${ }^{56}$ Biochemical tests were performed by using API 20E strips.

\section{Results}

The samples of blood and bone marrow were processed at The Aga Khan University Hospital's diagnostic laboratory. $S$ typhi was isolated from the blood $(n=58)$ or bone marrow $(n=88)$. The blood and bone marrow cultures of 12 patients showed no growth for any bacteria.

Thirty blood samples from 88 patients (whose bone marrow were positive) showed no bacterial growth. 


\section{Discussion}

Of the 100 patients, 88 had $S$ typhi in their blood or bone marrow, or both. This confirms our observation that $S$ typhi is a common cause of fever of unknown origin in Karachi. The remaining 12 patients whose blood or bone marrow cultures did not grow any organism recovered without any antibiotic treatment. This suggests that these patients probably had some viral infection(s).

In the patients with $S$ typhi infection (typhoid fever) the organism was isolated from the bone marrow of all of them. The organism was also isolated from the blood of $58(66 \%)$ patients. This means that bone marrow culture could confirm a diagnosis of typhoid fever in patients whose blood cultures are negative.

One of the reasons for blood cultures being negative and bone marrow being positive in typhoid fever may be antibiotic treatment before collection of blood for culture. An injudicious or suboptimal antibiotic regimen may diminish or eliminate the organism from blood, but $S$ typhi, being an intracellular organism, persists in the reticuloendothelial system including bone marrow. ${ }^{7}$ The intracellular location of bacteria protects them from conventional chemotherapeutic measures. ${ }^{89}$
We recommend that when typhoid fever is suspected blood for culture must be taken before antibiotics are given. In the case of patients who have already received antibiotics and whose blood cultures do not show any bacterial growth after three to four days of incubation, bone marrow should be cultured.

1 Farooqui BN, Ashfaq MK, Khurshid M, Ahmed MS Epidemiology of typhoid fever in Karachi. National Health 1990;8:20-1.

2 Hook EW. Salmonella species (including typhoid fever). In: Mandell GL, Douglas Jr RC, Bennett JE, eds. Principles and practice of infectious disease. New York: John Wiley Medical 1985: 1256-68.

3 Stuart BM, Pullen RZ. Typhoid: Clinical analysis of three hundred and sixty cases. Arch Intern Med 1946;78:629.

4 Robert H, Gilman, Terminat M, et al. Relative efficacy of blood, urine, rectal swab, bone marrow and rose-spot cultures of recovery of Salmonella typhi in typhoid fever. Lancet 1975; $1211-3$.

5 Rowe B, Hall M. Kauffman White Scheme. London: Central Health Laboratories, 1980:1-44.

6 Ewing WH. Antigenic scheme for Salmonella. In: Edwards and Ewing's Identification of Enterobacteriacae. 4th ed. and Ewing's Identification of Enterobacteriaca

7 Suter E. Interaction between phagocytes and pathogenic microorganisms. Bacteriol Rev 1956;20:94-132.

8 Mandell GL. Interaction of intraleukocytic bacteria and antibiotics. J Clin Invest 1973;52:1973-679.

9 Holmes B, Quie PG, Windhorst DP, Pollara B, Good RA Protection of phagocytized bacteria from the killing action of antibiotics. Nature 1966;210:1131-2.

\title{
Recovery of CD3 + and CD5 - lymphocyte subpopulation after autologous bone marrow transplantation and chemotherapy
}

\author{
P J McKay, A McLaren, L M Andrews, N P Lucie
}

\author{
Department of \\ Haematology, Western \\ Infirmary, Dumbarton \\ Road, Glasgow \\ G11 6NT \\ P J McKay \\ A McLaren \\ L M Andrews \\ N P Lucie \\ Correspondence to: \\ P J McKay \\ Accepted for publication \\ 13 September 1990
}

\begin{abstract}
Although most circulating $T$ cells in normal subjects express both $\mathrm{CD} 3$ and $\mathrm{CD} 5$ antigens on the cell surface, a small number lack the CD5 antigen. Recipients of allogeneic bone marrow transplants develop increased numbers of $\mathrm{CD} 3+$ CD5 - cells, particularly those who develop graft versus host disease (GVHD). This CD3 + CD5 - population may rise transiently in patients who have received an autologous bone marrow transplant (BMT) and in patients following completion of intensive chemotherapy for acute myeloid leukaemia (AML). These findings suggest that these CD3 + CD5 - cells are a normal component of the regenerating lymphoid system after BMT or chemotherapy.
\end{abstract}

The CD5 molecule is a 67000 molecular weight glycoprotein present on the surface of most human thymocytes and peripheral $T$ cells and on a subpopulation of $B$ cells. ${ }^{1-4} A$ small proportion of $\mathrm{CD} 3+\mathrm{T}$ cells in normal subjects do not express CD5. ${ }^{5}$ These cells are $\mathrm{CD} 2+, \mathrm{CD} 8+, \mathrm{CD} 4-$ and $\mathrm{CD} 16-$ and express low levels of CD7. ${ }^{5}$ They seem to be functional in that they have been shown to express normal cytolytic activity. ${ }^{5}$ An increase in these $\mathrm{CD} 3+\mathrm{CD} 5$ - cells has been reported after allogenic bone marrow transplantation, and a correlation has been found between the circulating CD $3+$ CDs - cells in the first 90 days after transplantation and the eventual development of all types of graft versus host disease (GVHD). ${ }^{6}$ In this study we measured $\mathrm{CD} 3+\mathrm{CD} 5$ - cells after autologous bone marrow transplantation and after drug treatment.

\section{Methods}

Samples of whole blood were collected in 